\title{
Clinical characteristics, biologic behavior, and survival after esophagectomy are similar for adenocarcinoma of the gastroesophageal junction and the distal esophagus
}

\author{
Jessica M. Leers, MD, ${ }^{\mathrm{a}}$ Steven R. DeMeester, MD, ${ }^{\mathrm{b}}$ Nadia Chan, MS, ${ }^{\mathrm{a}}$ Shahin Ayazi, MD, ${ }^{\mathrm{a}}$ \\ Arzu Oezcelik, MD, ${ }^{a}$ Emmanuele Abate, MD, ${ }^{a}$ Farzaneh Banki, MD, ${ }^{a}$ John C. Lipham, MD, ${ }^{a}$ \\ Jeffrey A. Hagen, MD, ${ }^{\mathrm{a}}$ and Tom R. DeMeester, $\mathrm{MD}^{\mathrm{a}}$
}

\begin{abstract}
Objective: The Siewert classification system differentiates between adenocarcinoma of the gastroesophageal junction and that of the distal esophagus. The purpose of this study was to evaluate whether there were differences in the location and prevalence of lymph node metastases, type of recurrence, and survival with these tumors that warrant distinguishing between them in clinical practice.
\end{abstract}

\begin{abstract}
Methods: Records of all patients who underwent resection for adenocarcinoma of the distal esophagus or gastroesophageal junction from 1987 to 2007 were retrospectively reviewed. Based on the endoscopic location of the epicenter of the tumor in relation to the gastroesophageal junction, tumors were categorized in 301 patients as being of the distal esophagus and in 208 as being of the gastroesophageal junction.
\end{abstract}

Results: There were no significant differences in age, sex, or body mass index between patients with adenocarcinoma of the distal esophagus or gastroesophageal junction. Patients with adenocarcinoma of the distal esophagus were more likely to have reflux symptoms $(75 \%$ vs $53 \%, P<.0001)$ and peritumoral intestinal metaplasia $(73 \%$ vs $51 \%, P<.0001)$ and be in a surveillance program $(54 \%$ vs $9 \%, P=.0005)$ compared with patients with adenocarcinoma of the gastroesophageal junction. However, the prevalence and location of nodal metastases was similar, and in node-positive patients mediastinal node involvement was present in more than $40 \%$ of the patients in each group (distal esophageal adenocarcinoma, 47\%; gastroesophageal junction adenocarcinoma, 41\%). Survival was similar (5 years: distal esophageal adenocarcinoma, $45 \%$; gastroesophageal junction adenocarcinoma, $38 \% ; P=.14)$, as was the prevalence and type of recurrence.

Conclusion: The prevalence and distribution of lymph node metastases in patients with adenocarcinoma of the distal esophagus and gastroesophageal junction were similar, and after esophagectomy, there was no difference in overall survival or recurrence. Efforts to differentiate between these tumors are unnecessary, and both are effectively treated with esophagectomy.

Earn CME credits at

http://cme.ctsnetjournals.org

The gastroesophageal junction (GEJ) represents the border between the esophagus and the stomach. Its perceived location differs based on whether external anatomic landmarks, endoscopic landmarks, or histology are used. ${ }^{1}$ Tumors at the GEJ are predominantly adenocarcinomas, and like adenocarcinoma of the distal esophagus (DE), the incidence of this cancer has been increasing rapidly over the past 2 decades in the Western

From the Departments of Surgery ${ }^{\mathrm{a}}$ and Cardiothoracic Surgery, ${ }^{\mathrm{b}}$ the University of Southern California, Keck School of Medicine, Los Angeles, Calif.

Received for publication May 9, 2008; revisions received April 16, 2009; accepted for publication May 22, 2009; available ahead of print July 15, 2009.

Address for reprints: Steven R. DeMeester, MD, 1510 San Pablo St, Suite 514, Los Angeles, CA, 90033 (E-mail: sdemeester@surgery.usc.edu).

J Thorac Cardiovasc Surg 2009;138:594-602

$0022-5223 / \$ 36.00$

Copyright (c) 2009 by The American Association for Thoracic Surgery doi:10.1016/j.jtcvs.2009.05.039 world. ${ }^{2}$ However, controversy and confusion persist regarding the location of the GEJ and the causes of tumors in this area.

In an effort to clarify some of the confusion and allow comparison of the results of treatment at different centers, Siewert and $\mathrm{Stein}^{3}$ introduced a system in which adenocarcinomas of the DE and proximal stomach were classified into 3 types based on the relationship of the epicenter of the tumor to the endoscopic location of the GEJ. Tumors with an epicenter 2 to $5 \mathrm{~cm}$ above the GEJ were considered type I or DE tumors, those within $2 \mathrm{~cm}$ (above or below) of the GEJ were type II or true GEJ tumors, and those with an epicenter 2 to $5 \mathrm{~cm}$ distal to the GEJ were type III or subcardial tumors (proximal gastric cancer). This classification system requires that the GEJ can be localized endoscopically, which can be difficult with large tumors, and implies that these cancers can be neatly categorized into those above, below, or at the GEJ. Furthermore, this classification system has been used to guide the selection of the surgical approach for tumors at different locations based on the belief that these tumors represent distinct clinical entities with different patterns of lymphatic spread and clinical outcome. ${ }^{4}$ Type I 


\section{Abbreviations and Acronyms \\ $\mathrm{CIM}=$ intestinal metaplasia of the cardia \\ $\mathrm{DE}=$ distal esophagus \\ GEJ = gastroesophageal junction}

tumors, which are considered esophageal in origin, are typically treated by means of an esophagectomy with abdominal and lower mediastinal node dissection. Type III tumors, which are considered to represent true gastric cancers, are typically treated with gastrectomy and resection of the regional abdominal lymph nodes. The management of type II (or GEJ) tumors remains less clear, with the debate centered on whether these are best treated like proximal gastric cancer or DE cancer. In addition, the location of potentially involved lymph nodes and the extent of lymphadenectomy necessary to adequately treat GEJ tumors remain disputed. Therefore the purpose of this study was to compare the prevalence and distribution of lymph node metastases, the prevalence and type of recurrence, and survival in patients with adenocarcinoma of the GEJ versus adenocarcinoma of the DE with the goal of determining whether an effort to distinguish between these tumors is warranted in clinical practice.

\section{MATERIALS AND METHODS}

We retrospectively reviewed the records of 613 patients who underwent resection for adenocarcinoma within $5 \mathrm{~cm}$ above or below the GEJ from January 1987 to June 2007. All patients had a preoperative upper endoscopy by a surgeon in the thoracic/foregut division, and during the endoscopy, the proximal and distal extents of the tumor and the location of the GEJ were noted. Assessment of the distal extent of the tumor included a retroflexed view to determine the degree of tumor extension below the GEJ. The GEJ was defined as the site where the proximal gastric rugal folds met the tubular esophagus with the stomach decompressed. The endoscopy report was used to retrospectively categorize tumors based on the Siewert classification system. Specifically, when the epicenter of the tumor was located 2 to $5 \mathrm{~cm}$ above the top of the gastric rugal folds, it was classified as a DE tumor, and when the epicenter was within $2 \mathrm{~cm}$ above or below the top of the gastric rugal folds, the tumor was classified as a GEJ tumor. By using this strategy, there were 301 patients with Siewert type I (or DE) adenocarcinoma and 208 with Siewert type II (or GEJ) adenocarcinoma. In 8 patients the tumor was classified as a proximal gastric cancer (Siewert Type III), and these patients were excluded. In 96 patients the size of the tumor precluded precise classification, and these patients were analyzed separately as an unclassified group. The study was approved by the Institutional Review Board of the Keck School of Medicine at the University of Southern California.

Preoperative evaluation included a detailed history focusing on the presence of reflux symptoms, standard laboratory tests, and a computed tomographic scan of the chest and abdomen. Endoscopic ultrasonographic analysis and positron emission tomographic scans were obtained later in this series as these tests became available. Neoadjuvant therapy was used selectively based on the local-regional extent of disease.

\section{Surgical Approach}

The operative approach was determined by the surgeon based on the location of the tumor, the patient's cardiopulmonary status, comorbid condi- tions, and the local-regional extent of disease. Our standard approach was an en bloc resection with 2-field lymphadenectomy. In some patients with intramucosal tumors, a vagal-sparing esophagectomy was performed, whereas a transhiatal or minimally invasive approach was used in patients with significant comorbid conditions or advanced age. A few patients with early GEJ tumors were treated with gastrectomy and a D2 lymph node dissection. The techniques for these procedures have been previously reported. ${ }^{2,5,6}$

\section{Histopathologic Assessment}

Hematoxylin and eosin-stained slides of paraffin-embedded tissue were used to determine tumor depth and the presence of lymph node metastases. Tumors were pathologically staged according to the TNM criteria for the esophagus by using the 6th edition of the Cancer Staging Manual published by the American Joint Committee on Cancer. Coexisting Barrett's esophagus was defined by the presence of intestinal metaplasia separate from the tumor mass either on preoperative endoscopic biopsy or in the surgical specimen.

\section{Follow-up}

All patients were routinely seen in the outpatient clinic after resection at intervals of 3 months for the first 3 years and every 6 months thereafter until 5 years. Beyond 5 years, follow-up visits were on an annual basis. Each visit included routine blood work and a computed tomographic scan of the chest and abdomen. Positron emission tomographic scans, once available, were obtained annually. Recurrent disease was diagnosed based on clinical, endoscopic, and/or radiographic findings, and histologic confirmation was sought when possible. Recurrence was classified as local-regional (occurring in the upper abdomen or mediastinum), distant (including all lymph node stations outside the lymphadenectomy field), and systemic (solid organ metastases). Follow-up was complete in 469 (92\%) of 509 patients until December 2007 or death.

\section{Statistical Analysis}

Data are presented as means unless otherwise noted. Proportions were compared by using Fisher's exact test, and means were compared by using the 2-sample Student's $t$ test or Mann-Whitney rank sum test. All-cause mortality and disease-specific survival curves were plotted by using the Kaplan-Meier method and compared by using the log-rank test. Univariate analysis was performed to identify factors that were different between patients with DE and GEJ tumors. Factors that were significantly different on univariate analysis were entered as covariates into a Cox regression analysis to compare survival and recurrence in patients with DE or GEJ adenocarcinoma adjusted for covariates.

\section{RESULTS}

Demographic information, type of resection, and pathologic findings in the 509 patients are shown in Table 1 . There were no significant differences in age, sex, or body mass index among patients with DE and GEJ tumors. Patients with DE adenocarcinoma were significantly more likely to have reflux symptoms and Barrett's esophagus and were more likely to have their tumors detected within a surveillance program compared with patients with GEJ adenocarcinoma. There were no significant differences in the median tumor length, type of resection, or use of neoadjuvant therapy. The TNM characteristics of the tumors are compared in Table 2. There was a higher prevalence of intramucosal tumors in the DE group compared with the GEJ group, likely related to the greater prevalence of surveillance endoscopy in the DE group. Correspondingly, there were more 
TABLE 1. Patient and tumor characteristics

\begin{tabular}{|c|c|c|c|}
\hline & $\begin{array}{l}\text { DE tumors } \\
(\mathrm{n}=301)\end{array}$ & $\begin{array}{c}\text { GEJ tumors } \\
(\mathbf{n}=\mathbf{2 0 8})\end{array}$ & $P$ value* \\
\hline Age in years $(\mathrm{SD})$ & $64.6( \pm 11.2)$ & $64.3( \pm 12.3)$ & .79 \\
\hline Male/female ratio & $6.8: 1$ & $6.5: 1$ & .67 \\
\hline $\begin{array}{l}\text { Body mass } \\
\text { index (SD) }\end{array}$ & $28.9( \pm 5.7)$ & $28.5( \pm 5.9)$ & .46 \\
\hline $\begin{array}{l}\text { History of heartburn or } \\
\text { regurgitation }\end{array}$ & $227(75.4 \%)$ & $114(54.8 \%)$ & $<.0001$ \\
\hline Barrett's surveillance & $62(20.6 \%)$ & $19(9.1 \%)$ & .0005 \\
\hline Presence of dysphagia & $162(53.8 \%)$ & $115(55.3 \%)$ & .79 \\
\hline $\begin{array}{l}\text { Presence of intestinal } \\
\text { metaplasia }\end{array}$ & $219(72.8 \%)$ & $106(51.0)$ & $<.0001$ \\
\hline Neoadjuvant therapy & $56(18.6 \%)$ & $38(18.3 \%)$ & 1.0 \\
\hline Type of resection & & & .69 \\
\hline $\begin{array}{l}\text { En bloc } \\
\quad \text { esophagectomy }\end{array}$ & $150(49.8 \%)$ & $100(48.1 \%)$ & \\
\hline $\begin{array}{l}\text { Transhiatal } \\
\text { esophagectomy }\end{array}$ & $110(36.5 \%)$ & $74(35.6 \%)$ & \\
\hline Other types & $41(13.6 \%)$ & $34(16.3 \%)$ & \\
\hline $\begin{array}{l}\text { Vagal-sparing } \\
\text { esophagectomy }\end{array}$ & $24(8.0 \%)$ & $14(6.7 \%)$ & \\
\hline $\begin{array}{l}\text { Transthoracic } \\
\text { esophagectomy }\end{array}$ & $17(5.6 \%)$ & $11(5.3 \%)$ & \\
\hline Total gastrectomy & 0 & $9(4.3 \%)$ & \\
\hline Length of tumor, $\mathrm{cm}(\mathrm{SD})$ & $3.2( \pm 2.6)$ & $3.4( \pm 2.6)$ & .29 \\
\hline \multicolumn{4}{|l|}{ Tumor differentiation } \\
\hline Well & $43(14.3 \%)$ & $32(15.4 \%)$ & .80 \\
\hline Moderate & $129(42.9 \%)$ & $77(37.0 \%)$ & .40 \\
\hline Poor & $129(42.9 \%)$ & $99(47.6 \%)$ & .52 \\
\hline
\end{tabular}

$D E$, Distal esophageal; GEJ, gastroesophageal junction; $S D$, standard deviation. *Mann-Whitney rank sum test for means and 2-sided Fisher's exact test for proportions.

transmural (T3 and T4) tumors in the GEJ group (GEJ group: $53 \%$ vs $\mathrm{DE}$ group: $43 \%, P=.06$ ). The prevalence of lymph node metastases and the number of involved lymph nodes was similar in patients with DE or GEJ adenocarcinoma. Table 3 shows the prevalence of lymph node involvement based on the depth of tumor invasion for both tumor locations. There was no significant difference in the prevalence of systemic disease found at the time of the operation or the completeness of resection. Greater than $90 \%$ of patients in both groups had an R0 resection.

There were 96 patients with adenocarcinomas too large or bulky to clearly assign to either the DE or GEJ group. The prevalence of a transmural tumor was significantly higher in this group (unclassified group: $69 \%$ vs DE group + GEJ group: $47 \%, P=.0005$ ), and significantly fewer patients in this group were node negative (unclassified group: $31 \%$ vs DE group + GEJ group: $47 \%, P=.004)$. Furthermore, patients with unclassified tumors had worse 5-year survival (unclassified group: $25 \%$ vs DE group + GEJ group: $42 \%$; $P=.02, \log$-rank test).

To accurately assess and compare the distribution of lymph node metastases in patients with DE and GEJ tumors, we analyzed only those patients who had a systematic thoracic and
TABLE 2. TNM classification

\begin{tabular}{|c|c|c|c|}
\hline & DE tumors & GEJ tumors & $P$ Value* \\
\hline \multicolumn{4}{|l|}{$\mathrm{T}$ classification } \\
\hline No neoadjuvant therapy & $\mathrm{n}=245$ & $\mathrm{n}=170$ & .20 \\
\hline $\mathrm{T} 1$ (intramucosal) & $71(23.6 \%)$ & $36(17.3 \%)$ & \\
\hline $\mathrm{T} 1$ (submucosal) & $38(12.6 \%)$ & $26(12.5 \%)$ & \\
\hline $\mathrm{T} 2$ & $30(10.0 \%)$ & $18(8.7 \%)$ & \\
\hline $\mathrm{T} 3$ & $104(34.5 \%)$ & $82(39.4 \%)$ & \\
\hline $\mathrm{T} 4 \dagger$ & $2(0.7 \%)$ & $8(3.8 \%)$ & \\
\hline $\begin{array}{l}\mathrm{N} \text { classification } \\
\quad( \pm \text { neoadjuvant })\end{array}$ & $\mathrm{n}=301$ & $\mathrm{n}=208$ & \\
\hline No & $151(50.2 \%)$ & $90(43.3 \%)$ & .18 \\
\hline N1 & $150(49.8 \%)$ & $118(56.7 \%)$ & \\
\hline \multicolumn{4}{|l|}{ No. of nodes involved } \\
\hline $1-4$ & $66(21.9 \%)$ & $50(22.1 \%)$ & .74 \\
\hline $5-8$ & $24(8.0 \%)$ & $22(11.1 \%)$ & \\
\hline$>8$ & $61(20.3 \%)$ & $42(21.1 \%)$ & \\
\hline Metastasis M1b & $12(3.9 \%)$ & $9(4.5 \%)$ & .83 \\
\hline Overall stage & & & .52 \\
\hline No neoadjuvant therapy & $\mathrm{n}=245$ & $\mathrm{n}=170$ & \\
\hline I & $96(31.9 \%)$ & $57(27.4 \%)$ & \\
\hline IIa & $26(8.6 \%)$ & $16(7.7 \%)$ & \\
\hline $\mathrm{IIb}$ & $25(8.3 \%)$ & $15(7.2 \%)$ & \\
\hline III & $88(29.2 \%)$ & $76(36.5 \%)$ & \\
\hline IV & $10(3.3 \%)$ & $6(2.9 \%)$ & \\
\hline Neoadjuvant therapy & $56(18.6 \%)$ & $38(18.3 \%)$ & 1.0 \\
\hline $\begin{array}{l}\text { Complete pathologic } \\
\text { response }\end{array}$ & $12(21.4 \%)$ & $6(15.8 \%)$ & .59 \\
\hline \multicolumn{4}{|l|}{$\mathrm{R}$ category } \\
\hline R0 & $278(92.4 \%)$ & $193(92.7 \%)$ & 1.0 \\
\hline $\mathrm{R} 1 / 2$ & $23(7.6 \%)$ & $15(7.3 \%)$ & \\
\hline \multicolumn{4}{|l|}{$\begin{array}{l}\text { Location of } \\
\text { positive margin }\end{array}$} \\
\hline Gastric staple line & 4 & 3 & \\
\hline Proximal esophageal & 4 & 1 & \\
\hline Radial & 8 & 7 & \\
\hline Diaphragm & 4 & 2 & \\
\hline Other & 3 & 2 & \\
\hline
\end{tabular}

$D E$, Distal esophageal; GEJ, gastroesophageal junction. *Two-sided Fisher's exact test or $\chi^{2}$ test. $\dagger$ Reason for T4: involvement of perigastric fat, diaphragm, pericardium, or distal pancreas.

abdominal lymphadenectomy (en bloc resection) without neoadjuvant therapy. The prevalence of lymph node involvement at each location is shown in Table 4. The pattern of lymph node metastases in patients with $\mathrm{N} 1$ disease who had an en bloc esophagectomy is shown in Figure 1. The prevalence of node involvement and the distribution of involved lymph nodes were similar for DE and GEJ tumors. Importantly, more than $40 \%$ of node-positive patients had involved nodes in the mediastinum whether the tumor was located in the DE or at the GEJ. In $9 \%$ of patients with DE tumors and $8 \%$ of patients with GEJ tumors, a positive mediastinal node was the only site of lymph node involvement (all resected abdominal lymph nodes were negative).

The median follow-up in surviving patients was 37 months (interquartile range, $8-46$ months) and did not differ between patients with DE and GEJ tumors (38 months and 
TABLE 3. The prevalence of lymph node metastases based on tumor depth of invasion (patients with neoadjuvant therapy excluded)

\begin{tabular}{lccc}
\hline $\begin{array}{c}\text { Depth of } \\
\text { invasion }\end{array}$ & $\begin{array}{c}\text { DE tumors } \\
(\mathbf{n}=\mathbf{2 4 5})\end{array}$ & $\begin{array}{c}\text { GEJ tumors } \\
(\mathbf{n}=\mathbf{1 7 2})\end{array}$ & $\boldsymbol{P}$ value* \\
\hline Intramucosal & $2 / 71(2.8 \%)$ & $1 / 36(2.8 \%)$ & 1.0 \\
Submucosal & $11 / 38(28.9 \%)$ & $6 / 29(20.7 \%)$ & .59 \\
Intramural & $15 / 30(50.0 \%)$ & $8 / 16(50.0 \%)$ & 1.0 \\
Transmural & $96 / 106(90.6 \%)$ & $79 / 91(86.8 \%)$ & .92 \\
\hline
\end{tabular}

$D E$, Distal esophageal; $G E J$, gastroesophageal junction. *Two-sided Fisher's exact test.

36 months, $P=.64)$. Overall and disease-specific survival was similar for patients with DE and GEJ tumors (Figure 2). Survival at 5 and 10 years was $45 \%$ and $25 \%$ for patients with DE tumors compared with $38 \%$ and $31 \%$ for patients with GEJ tumors, respectively. The 5- and 10-year survivals for both tumor locations considered together are $42 \%$ and $27 \%$, respectively. A Cox proportional hazard model was used to compare survival in patients with GEJ or DE adenocarcinoma adjusted for covariates found to be significant on univariate analysis in Tables 1 and 2 (history of heartburn or regurgitation, Barrett's surveillance, and presence of intestinal metaplasia). Cox regression analysis showed no significant difference in survival between patients with GEJ adenocarcinoma and those with DE adenocarcinoma (adjusted hazard ratio [GEJ vs DE], 1.04; 95\% confidence interval, $0.81-1.34 ; P=.78$ ).

TABLE 4. Prevalence and location of lymph node metastases in patients who had en bloc resection (all patients not having en bloc resection were excluded)

\begin{tabular}{lccc}
\hline & $\begin{array}{c}\text { DE tumors } \\
(\mathbf{n}=\mathbf{1 5 0})\end{array}$ & $\begin{array}{c}\text { GEJ tumors } \\
(\mathbf{n}=\mathbf{1 0 0})\end{array}$ & $\boldsymbol{P}$ value* \\
\hline Prevalence of N1 disease & $83(55 \%)$ & $61(61 \%)$ & .43 \\
Location of positive node(s) & & & \\
$\quad$ Mediastinal & $39(26 \%)$ & $25(25 \%)$ & .88 \\
$\quad$ Paratracheal & $3(2 \%)$ & 0 & \\
$\quad$ Subcarinal & $12(8 \%)$ & $3(3 \%)$ & \\
$\quad$ Paraesophageal & $38(25 \%)$ & $25(25 \%)$ & \\
Abdominal & $70(47 \%)$ & $52(52 \%)$ & .44 \\
$\quad$ Parahiatal & $33(22 \%)$ & $20(20 \%)$ & \\
$\quad$ Perigastric & $52(35 \%)$ & $45(45 \%)$ & \\
Celiac & $9(6 \%)$ & $3(3 \%)$ & \\
Other & $13(9 \%)$ & $8(8 \%)$ & \\
\hline
\end{tabular}

Values are presented as numbers with percentages as shown. $D E$, Distal esophageal $G E J$, gastroesophageal junction. *Two-sided Fisher's exact test.

Recurrent cancer was found in 124 (41\%) patients with DE tumors and in $81(39 \%)$ patients with GEJ tumors $(P=.65)$. The type of recurrence was similar for both tumor locations, with the majority being systemic (Table 5). Carcinomatosis was uncommon and not significantly different between groups $(P=.13)$. A Cox proportional hazard model was used to compare recurrence in patients with GEJ or DE adenocarcinoma adjusted for covariates found to be
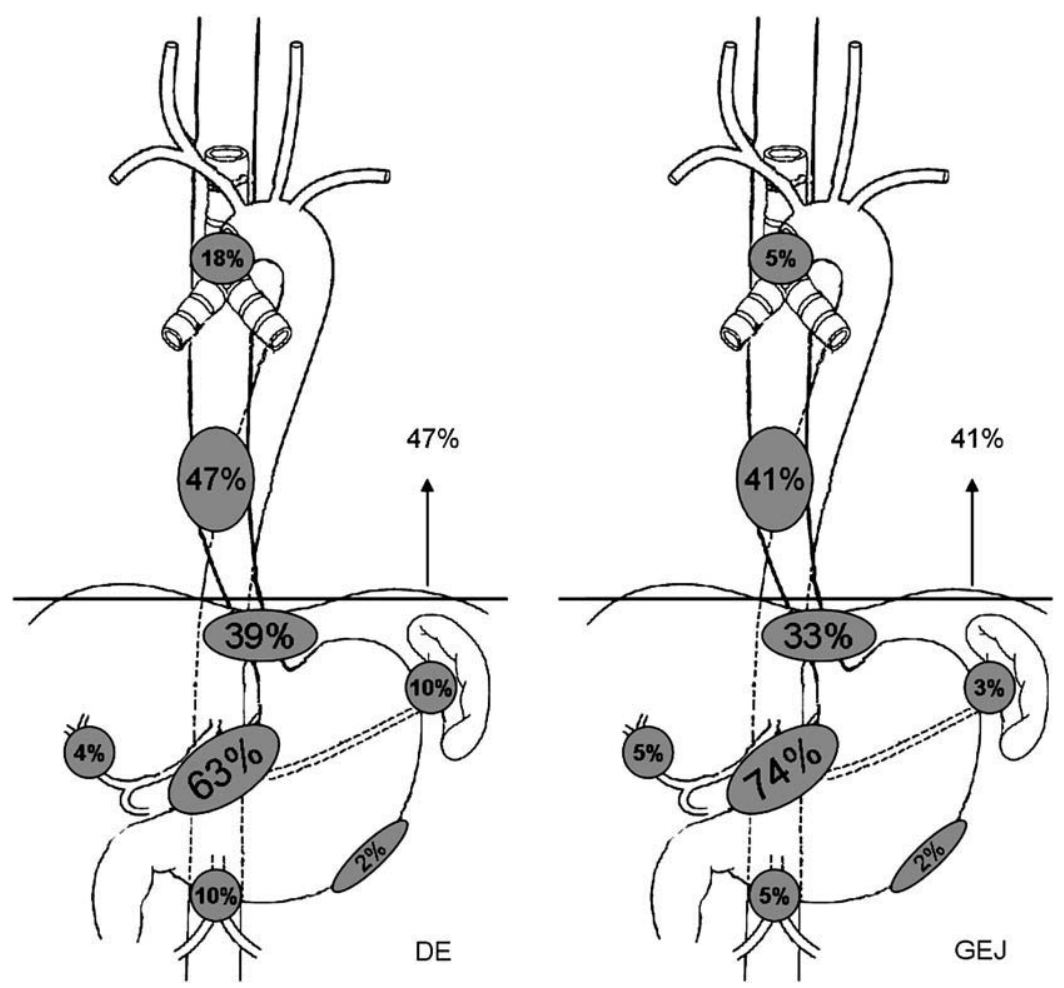

FIGURE 1. The pattern of lymph node involvement in patients with $\mathrm{N} 1$ disease after en bloc esophagectomy $(\mathrm{n}=144)$ with distal esophageal $(D E)$ and gastroesophageal junction $(G E J)$ tumors. 

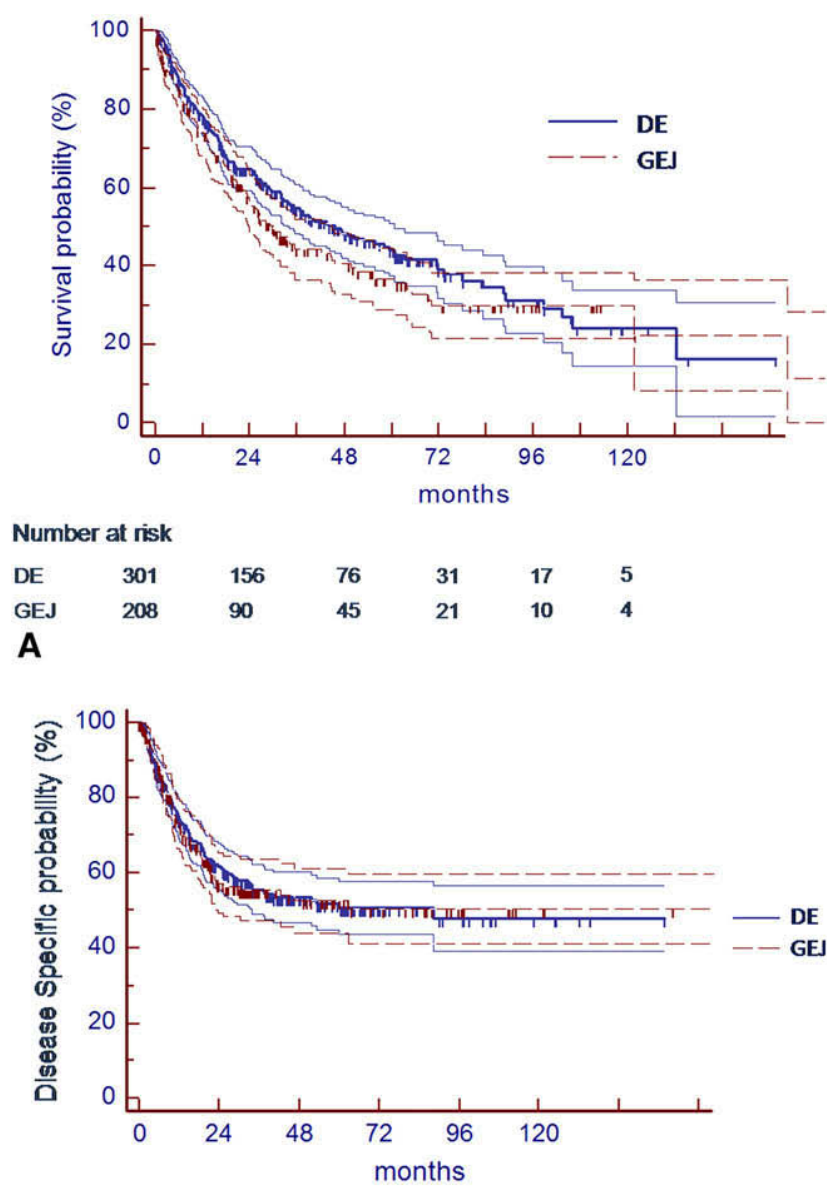

Number at risk

$\begin{array}{lllllll}\text { DE } & 289 & 123 & 63 & 26 & 15 & 5 \\ \text { GEJ } & 199 & 66 & 38 & 21 & 10 & 4\end{array}$

B

FIGURE 2. A, Five-year overall survival curve for patients with distal esophageal $(D E)$ and gastroesophageal junction $(G E J)$ adenocarcinomas ( $P=.16, \log$-rank test). B, Five-year disease-specific survival for patients with distal esophageal $(D E)$ and gastroesophageal junction $(G E J)$ adenocarcinomas $(P=.41, \log$-rank test $)$.

significant on univariate analysis in Tables 1 and 2 (history of heartburn or regurgitation, Barrett's surveillance, and presence of intestinal metaplasia). Cox regression analysis showed no significant difference in recurrence for patients with GEJ adenocarcinoma or those with DE adenocarcinoma (adjusted hazard ratio [GEJ vs DE], 0.82; 95\% confidence interval, $0.61-1.10 ; P=.19)$. The majority of patients who survived beyond 5 years died from a noncancer cause.

\section{DISCUSSION}

In contrast to distal gastric cancers, the incidence of adenocarcinoma of both the GEJ and DE has increased dramatically over the past 25 years in the United States. ${ }^{2}$ Esophageal adenocarcinoma develops secondary to chronic gastroesophageal reflux disease and Barrett's esophagus.
TABLE 5. Prevalence and site of recurrence (patients might have more than 1 site of recurrence)

\begin{tabular}{lccc}
\hline & DE tumors & GEJ tumors & $P$ value* \\
\hline Surgical intervention alone & $\mathrm{n}=245$ & $\mathrm{n}=170$ & \\
Any recurrence & $93(38.0 \%)$ & $60(35.6 \%)$ & .61 \\
Site of recurrence & & & \\
Locoregional & $17(6.9 \%)$ & $12(7.1 \%)$ & \\
Distant nodes & $28(11.4 \%)$ & $13(7.6 \%)$ & \\
Systemic & $63(25.7 \%)$ & $42(24.7 \%)$ & \\
Liver & 31 & 21 & \\
Lung & 16 & 9 & \\
Bone & 10 & 6 & \\
Brain & 3 & 3 & \\
Carcinomatosis & 2 & 5 & \\
Skin & 2 & 1.0 \\
$\quad$ Other & 6 & 3 & \\
Neoadjuvant therapy + & $\mathrm{n}=56$ & $\mathrm{n}=38$ & \\
surgical intervention & & & \\
Any recurrence* & $31(55.4 \%)$ & $21(55.3 \%)$ \\
Site of recurrence & & & \\
Locoregional & $4(7.1 \%)$ & $3(7.9 \%)$ & \\
Distant nodes & $7(12.5 \%)$ & $2(5.3 \%)$ & \\
Systemic & $21(37.5 \%)$ & $18(47.4 \%)$ & \\
\hline$D E$ Distal esophageal; GEJ, gastroesophageal junction. *Two-sided Fisher's exact \\
test.
\end{tabular}

The cause of adenocarcinoma of the GEJ is less clear, but there is increasing evidence that it also follows a path from intestinal metaplasia to dysplasia and cancer. ${ }^{1,7,8}$ It is well established that intestinal metaplasia is a common pathologic finding when biopsy specimens are obtained at an endoscopically normal-appearing GEJ, and in some patients dysplasia and small cancers have been found confined to the GEJ, confirming the link between intestinal metaplasia of the cardia (CIM) and adenocarcinoma of the GEJ. ${ }^{8,9}$ It is likely that in some patients CIM develops as a consequence of Helicobacter pylori infection and pangastric intestinal metaplasia, whereas in others with no intestinal metaplasia in the antrum and no history of $H$ pylori infection, CIM is likely secondary to a reflux cause. Although CIM appears to have a lower malignant potential than Barrett's esophagus, this lower malignant potential is offset by the greater prevalence of CIM in the population. ${ }^{1,9}$

The GEJ remains a controversial area, with even the normal mucosa at this site disputed. ${ }^{10-13}$ Furthermore, the presumed location of the GEJ varies depending on whether it is being viewed endoscopically, surgically, or pathologically. Most commonly, the GEJ is seen endoscopically, and the accepted definition is the site where the proximal limits of the gastric rugal folds in a nondistended stomach meet the tubular esophagus. However, in patients with a DE cancer, determining the precise location of the GEJ can be difficult. This difficulty was illustrated in a recent prospective randomized trial on esophageal and GEJ adenocarcinoma from The Netherlands, in which the authors noted 
that "a substantial difference existed between the endoscopic tumor classification, which was used for the preoperative stratification process, and the pathologic tumor classification in the resected specimen."14 They reported that frequently what the endoscopist labeled as a DE adenocarcinoma, the pathologist considered a GEJ tumor.

The difficulty with endoscopic determination of the GEJ has also been shown in a recent autopsy study by Chandrasoma and colleagues. ${ }^{15}$ In this study the authors found that the reflux-damaged DE can become dilated, develop rugal folds, and be lined by columnar mucosa, thereby mimicking the proximal stomach. They used the histologic presence of submucosal glands and the location of gastric oxyntic mucosa to conclude that the true GEJ was as much as 2 $\mathrm{cm}$ distal to the endoscopically determined location. Given the inaccuracy of the endoscopic determination of the GEJ, Chandrasoma and colleagues proposed that GEJ tumors should be classified based on the type of epithelium at the epicenter of the tumor and at the distal margin of the tumor rather than the perceived relationship of the epicenter of the tumor to the endoscopically determined location of the GEJ. Using this method, they found that $30(83 \%)$ of 36 adenocarcinomas that were considered to be GEJ tumors were in fact DE cancers.

Another problem with trying to relate the epicenter of a tumor with the location of the GEJ is that large tumors often obscure the landmarks to the point that a clear determination of whether a tumor is located in the DE or at the GEJ is not possible, as was the case in 96 patients in this study. Given the frequent discrepancy between the endoscopic and pathologic location of the GEJ and the problem with large tumors obscuring the commonly used landmarks, we wondered whether an effort to distinguish between adenocarcinoma of the DE and the GEJ was clinically necessary and undertook a review of our experience with these tumors. We found that although there were differences in symptoms, the presence of intestinal metaplasia, and involvement in a surveillance endoscopy program between patients with adenocarcinoma of the DE and those with adenocarcinoma of the GEJ, there were no significant differences in the prevalence or location of nodal metastases, type of recurrence, or overall or disease-specific survival in patients with these tumors.

An important aspect of this study is that the majority of patients $(>95 \%)$ were treated similarly with an esophagectomy, and most commonly, an en bloc resection was performed. Other series typically include a high percentage of total gastrectomies for GEJ adenocarcinoma, and this compromises assessment of the pattern and location of node metastases. In the series by Siewert and associates, ${ }^{4}$ lower mediastinal nodes were involved in $15.6 \%$ of patients. Subcarinal nodal involvement was not specified, likely because most patients $(82 \%)$ with GEJ tumors at their center were treated with extended gastrectomy rather than esophagec- tomy. In this study we compared the prevalence and pattern of node metastases only in patients who had an en bloc resection with systematic mediastinal and upper abdominal lymphadenectomy, and we were therefore able to definitively compare the prevalence and distribution of lymph node metastases in patients with DE or GEJ adenocarcinomas. We found that mediastinal nodal involvement was present in $26 \%$ of DE adenocarcinomas and $25 \%$ of GEJ adenocarcinomas, with the majority of involved nodes located in the paraesophageal region for both tumor locations. Furthermore, abdominal node involvement was present in $47 \%$ of DE adenocarcinomas and $52 \%$ of GEJ adenocarcinomas, with the majority of involved nodes located along the lesser curvature for both tumor locations. Importantly, when only patients with at least 1 involved node were considered (patients with $\mathrm{N} 1$ disease), we found that $47 \%$ of patients with DE adenocarcinomas and $41 \%$ of patients with GEJ adenocarcinomas had a positive mediastinal node, and in $9 \%$ and $8 \%$, respectively, this was the only site of nodal involvement.

Given the high prevalence of mediastinal node involvement with both DE and GEJ adenocarcinomas, it is clear that a lower mediastinal node dissection needs to be included in the surgical therapy for both of these tumors. This fact is acknowledged in the study by Siewert and associates, ${ }^{4}$ who routinely split the diaphragm and resect the lower mediastinal nodes as part of a total gastrectomy for tumors at the GEJ. ${ }^{16}$ Had this not been part of the operative strategy, it is unlikely that they would have shown equivalent survival for esophagectomy and total gastrectomy in patients with adenocarcinomas located at the GEJ. The danger with recommending gastrectomy for GEJ adenocarcinomas is that few centers have the experience that Siewert and associates do, and the tendency among low-volume centers is likely to be to minimize the mediastinal dissection and perhaps compromise on the esophageal resection margin. Although the frequency of a positive proximal resection margin for patients with GEJ tumors who had a total gastrectomy in the series by Siewert and associates is not specified, the article indicates that an R0 resection was only accomplished in $75 \%$ of patients. ${ }^{4}$ This is substantially lower than our R0 resection rate of greater than $90 \%$ when esophagectomy was the preferred resection technique. In keeping with concerns regarding the proximal resection margin obtained when a gastrectomy rather than an esophagectomy is performed for GEJ adenocarcinoma, Barbour and coworkers ${ }^{17}$ from the Memorial Sloan-Kettering cancer center recently reported that survival was significantly improved when the gross esophageal resection margin was greater than $3.8 \mathrm{~cm}$ from the tumor in the fixed specimen (which translated to $5 \mathrm{~cm}$ in the native state). This was best accomplished with an esophagectomy, and the authors noted significantly improved survival in patients who had an esophagectomy for GEJ adenocarcinomas compared with those who had 
a gastrectomy. Interestingly, in their analysis they included all Siewert types (I, II, and III), and it is likely that if the analysis had been restricted to only Siewert types I and II (excluding the subcardial or proximal gastric cancers), the significance in favor of esophagectomy over gastrectomy would have been even more striking.

We favor an en bloc resection in physiologically suitable patients with tumors of the DE or GEJ and have reported good survival and rare local recurrence with this procedure. Previously, we retrospectively reviewed our experience with transhiatal versus en bloc resection in patients with a DE or GEJ T3 N1 adenocarcinoma and reported that survival was significantly better with the en bloc procedure when there were 8 or fewer involved nodes. ${ }^{18}$ Beyond 8 involved nodes, survival was poor and independent of the type of resection. Our retrospective results have recently been confirmed in a randomized prospective trial of en bloc transthoracic versus transhiatal esophagectomy for adenocarcinoma of the DE. ${ }^{14}$ Subgroup analysis in this trial showed a statistically significant $20 \%$ improvement in survival for patients who had an en bloc resection compared with those who had a transhiatal esophagectomy when there were 1 to 8 involved nodes.

We have also shown that the benefit of an en bloc resection remains evident even after neoadjuvant therapy. We evaluated survival in patients with residual disease after neoadjuvant therapy and reported a significant improvement in survival for those patients with adenocarcinoma of the DE or GEJ who had an en bloc compared with a transhiatal resection. ${ }^{19}$ The explanation for the improved survival after en bloc esophagectomy is in part related to the improved local control that the en bloc resection provides. However, there also seems to be a direct benefit from the thorough lymphadenectomy that accompanies an en bloc resection. Using pooled data from 9 international esophageal cancer centers, we have shown that the number of lymph nodes removed with an esophagectomy is an independent predictor of survival and that the likelihood of survival is maximized when a minimum of 23 lymph nodes are resected..$^{20}$ Similar findings supporting the role for extended lymphadenectomy in patients with esophageal cancer have been published by Altorki and colleagues $^{21}$ and Schwarz and Smith. ${ }^{22}$ Even in node-negative patients a positive effect on survival has been demonstrated with a more extensive lymphadenectomy. ${ }^{23}$ These results support the routine use of an en bloc esophagectomy in patients with both GEJ and DE adenocarcinomas.

In the current study we have shown that the prevalence and type of recurrence was similar for both GEJ and DE adenocarcinomas. Most recurrences were systemic, with a low rate of abdominal carcinomatosis for both tumor locations. In the patients who had neoadjuvant therapy in this series, we did not see a clear reduction in local-regional recurrence rates, perhaps because of our frequent use of the en bloc procedure. Likewise, systemic disease was the predominant form of recurrence with and without neoadjuvant therapy. Improved therapies to control systemic metastases with this disease are critical to building on the improvements in local control that have been achieved with the en bloc surgical technique. We found that overall and disease-specific survival were similar for adenocarcinoma of the DE and GEJ and showed that both tumors are effectively treated with esophagectomy. Thus from a clinical standpoint, our study and others suggest that efforts to preoperatively determine whether the tumor is a DE adenocarcinoma or a GEJ adenocarcinoma are unnecessary. ${ }^{24}$

Our study is limited by the fact that it is retrospective, although this limitation is minimized by the fact that all patients were treated in a single high-volume center by experienced esophageal surgeons. Furthermore, the surgical technique and our philosophy regarding treatment for these tumors are standardized, with $100 \%$ of patients with adenocarcinoma of the DE and more than $95 \%$ of patients with adenocarcinoma of the GEJ having an esophagectomy. Nearly $50 \%$ of patients in each group had an en bloc esophagectomy, and this allowed us to accurately determine the prevalence and distribution of lymph node metastases for tumors in each location.

Another limitation is that there were $96(19 \%)$ patients with tumors so large and bulky that we were unable to categorize them as DE or GEJ in origin. However, this underlines the problem with the Siewert classification system and reflects the reality that we face as clinicians. By demonstrating that it is not necessary to determine whether an adenocarcinoma near the GEJ is a DE or a GEJ cancer, we simplify the management of these patients and avoid the ambiguity that is often present because of variable and indistinct endoscopic landmarks and the presence of large tumors that are not readily classifiable.

Lastly, a potential weakness is that we were unable to compare the pathologic determination of the location of the tumor with our endoscopic determination because our pathologists did not assess tumor location at the time of specimen resection in the operating room. However, a determination of the location of a tumor that can only be accurately made on pathologic examination of the resected specimen would not be clinically useful for guiding the choice of therapy in these patients. Therefore we believe that focusing our analysis on the preoperative endoscopic assessment of tumor location was the most appropriate methodology for this study.

In conclusion, we have shown that the clinically relevant issues related to the therapy of adenocarcinoma of the GEJ or DE, including the prevalence and distribution of node metastases, the prevalence and type of recurrence, and overall and disease-specific survival, are similar for these tumors. The high prevalence of nodal metastases in the lower mediastinum makes a lymphadenectomy in this region a critical part of successful surgical therapy for both tumors and 
supports the routine use of esophagectomy for these patients. There are inherent inaccuracies in trying to determine the precise location of the GEJ and the relationship of the epicenter of a cancer to the GEJ. Given these difficulties and the lack of a significant difference in the biologic behavior between adenocarcinoma of the DE and GEJ, we suggest that efforts to determine the precise origin of the tumor are not necessary and that an esophagectomy, preferably an en bloc resection, is appropriate surgical therapy for adenocarcinoma in either location.

We thank Linda Chan from the University of Southern California for her dedicated support and advice regarding statistical analysis and methodology in this article.

\section{References}

1. DeMeester SR, DeMeester TR. Columnar mucosa and intestinal metaplasia of the esophagus: fifty years of controversy. Ann Surg. 2000;231:303-21.

2. DeMeester SR. Adenocarcinoma of the esophagus and cardia: a review of the disease and its treatment. Ann Surg Oncol. 2006;13:12-30.

3. Siewert JR, Stein HJ. Classification of adenocarcinoma of the oesophagogastric junction. Br J Surg. 1998;85:1457-9.

4. Siewert RJ, Feith M, Werner M, Stein HJ. Adenocarcinoma of the esophagogastric junction: results of surgical therapy based on anatomical/topographic classification in 1,002 consecutive patients. Ann Surg. 2000;232:353-61.

5. DeMeester SR. Endoscopic mucosal resection and vagal-sparing esophagectomy for high-grade dysplasia and adenocarcinoma of the esophagus. Semin Thorac Cardiovasc Surg. 2005; 17:320-5.

6. Hagen JA, DeMeester SR, Peters JH, Chandrasoma P, DeMeester TR. Curative resection for esophageal adenocarcinoma: analysis of 100 en bloc esophagectomies. Ann Surg. 2001;234:520-31

7. Ruol A, Parenti A, Zaninotto G, Merigliano S, Costantini M, Cagol M, et al. Intestinal metaplasia is the probable common precursor of adenocarcinoma in Barrett esophagus and adenocarcinoma of the gastric cardia. Cancer. 2000;88:2520-8.

8. Cameron AJ. Epidemiology of Barrett's esophagus and adenocarcinoma. Dis Esophagus. 2002;15:106-8.

9. Guelrud M, Herrera I, Essenfeld H, Castro J, Antonioli DA. Intestinal metaplasia of the gastric cardia: a prospective study with enhanced magnification endoscopy. Am J Gastroenterol. 2002;97:584-9.

10. Chandrasoma P, Makarewicz K, Wickramasinghe K, Ma Y, Demeester T. A proposal for a new validated histological definition of the gastroesophageal junction. Hum Pathol. 2006;37:40-7.

11. Chandrasoma PT, Der R, Ma Y, Dalton P, Taira M. Histology of the gastroesophageal junction: an autopsy study. Am J Surg Pathol. 2000;24:402-9.

12. Ormsby AH, Kilgore SP, Goldblum JR, Richter JE, Rice TW, Gramlich TL. The location and frequency of intestinal metaplasia at the esophagogastric junction in 223 consecutive autopsies: implications for patient treatment and preventive strategies in Barrett's esophagus. Mod Pathol. 2000;13:614-20.

13. Chandrasoma P. Controversies of the cardiac mucosa and Barrett's oesophagus. Histopathology. 2005;46:361-73.

14. Omloo JM, Lagarde SM, Hulscher JB, Reitsma JB, Fockens P, van Dekken H, et al. Extended transthoracic resection compared with limited transhiatal resection for adenocarcinoma of the mid/distal esophagus: five-year survival of a randomized clinical trial. Ann Surg. 2007;246:992-1001.

15. Chandrasoma P, Wickramasinghe K, Ma Y, DeMeester T. Adenocarcinomas of the distal esophagus and "gastric cardia" are predominantly esophageal carcinomas. Am J Surg Pathol. 2007;31:569-75.

16. Stein HJ, Feith M, Siewert JR. Cancer of the esophagogastric junction. Surg Oncol. 2000;9:35-41.

17. Barbour AP, Rizk NP, Gonen M, Tang L, Bains MS, Rusch VW, et al. Adenocarcinoma of the gastroesophageal junction: influence of esophageal resection margin and operative approach on outcome. Ann Surg. 2007;246:1-8.

18. Johansson J, DeMeester TR, Hagen JA, DeMeester SR, Peters JH, Oberg S, et al. En bloc vs transhiatal esophagectomy for stage T3 N1 adenocarcinoma of the distal esophagus. Arch Surg. 2004;139:627-33.

19. Rizetto C, DeMeester SR, Hagen JA, Peyre CG, Lipham JC, DeMeester TR. En bloc esophagectomy reduces local recurrence and improves survival compared with transhiatal resection after neoadjuvant therapy for esophageal adenocarcinoma. J Thorac Cardiovasc Surg. 2008;135:1228-36.

20. Peyre CG, Hagen JA, DeMeester SR, Altorki NK, Ancona E, Griffin MS, et al. The number of lymph nodes removed predicts survival in esophageal cancer: an international study on the impact of extent of surgical resection. Ann Surg. 2008;248:549-56.

21. Altorki NK, Zhou XK, Stiles B, Port JL, Paul S, Lee PC, et al. Total number of resected lymph nodes predicts survival in esophageal cancer. Ann Surg. 2008; 248:221-6.

22. Schwarz RE, Smith DD. Clinical impact of lymphadenectomy extent in resectable esophageal cancer. J Gastrointest Surg. 2007;11:1384-94.

23. Greenstein AJ, Litle VR, Swanson SJ, Divino CM, Packer S, Wisnivesky JP. Effect of the number of lymph nodes sampled on postoperative survival of lymph node-negative esophageal cancer. Cancer. 2008;112:1239-46.

24. de Manzoni G, Pedrazzani C, Pasini F, Di Leo A, Durante E, Castaldini G, et al. Results of surgical treatment of adenocarcinoma of the gastric cardia. Ann Thorac Surg. 2002;73:1035-40.

\section{Discussion}

Dr Thomas W. Rice (Cleveland, Ohio). Confusion and controversy surrounds the esophagogastric junction. I would like to thank Dr Leers and her colleagues for presenting this most important analysis of their experience with the surgical treatment of cancers of the esophagogastric junction. It is reports such as this that will shed light on this contentious transition area of the upper gastrointestinal tract, help end therapeutic disputes, and guide us in the treatment of cancers arising in this borderland.

This 10-cm/4-inch segment of the DE and proximal stomach has been classified into 3 areas by Dr Siewert and his colleagues. Importantly, their classification is based on pathologic assessment of the epicenter of the tumor, an easy thing to do in the quiet of the pathology laboratory. However, if this information is to be clinically important, it must be available before treatment in the living, swallowing world of the esophagoscopy suite. Dr Leers and her colleagues rightly determined Siewert type at initial endoscopy. They point out the difficulty of defining the esophagogastric junction endoscopically but have rigorously attempted to type these cancers in 613 patients. Not surprisingly, every sixth patient defied classification. At first, I wondered how these missing data should have been handled in the analysis, but it became evident that these 96 unclassifiable patients represented an interesting quasi-control group for your analysis. Although it might require propensity scoring, because these are typically larger tumors, it would strengthen your argument if patients with unclassifiable tumors had a survival similar to that of patients from either classifiable group.

My first question is this: Have you or will you add this group to your analysis?

Dr DeMeester. Thanks, Tom. Yes, we actually did that. The same thought struck us, so we plotted the Kaplan-Meier survival for those patients, and it is identical. It actually falls right in between the 2 lines for DE and GEJ tumors. Therefore we looked at that. There was some debate within our group as to whether it was legitimate to include those patients because, as we said, we could not really fit them into either the DE or GEJ group, and whether their addition just muddied the waters was a concern. Therefore the final decision was to leave them out. But it is certainly an interesting point, and at least now in the discussion it will be evident to everybody that the survival for these unclassified tumors falls right in between the lines for the other 2 groups. 
Dr Rice. Siewert and associates have demonstrated that as Siewert tumor type increases from I to II to III, cancers have a higher T, a higher $\mathrm{N}$, a higher $\mathrm{M}$, higher histologic grade, and more cancer-positive nodes. Although your data only include two thirds of this classification schema, if you look carefully, type II cancers compared with type I cancers have a significantly higher T, $7 \%$ more N1, and $4 \%$ of cancers with 5 or more positive lymph nodes, and at 5 years, there is a 7\% difference in survival. You claim that these groups are similar, but they are systematically slightly different, and these differences might be masked to some extent by the group composition.

I have 2 questions. Have you considered the addition of histologic grade, as has Siewert, that would allow for more equal group comparisons? And to permit a fair comparison of these retrospective data, have you considered matching patients, such as with propensity scores, allowing risk adjustment and therefore analysis of comparable groups that differ only by the Siewert tumor type?

Dr DeMeester. It is an interesting issue. The debate about whether tumor grade is important or not is really, I think, coming to the forefront when you look at early tumors. Ell and colleagues suggested that well-differentiated tumors should be treated differently than poorly differentiated tumors. In my own practice, when you have a reasonably sized mass, it has not made a difference, and at this point, I do not factor tumor grade in when I do endoscopic mucosal resection as therapy for these early tumors. Therefore it is not clear that it is an important distinction, although it is something to consider.

In terms of further forms of analysis, I think that we could certainly do some type of matching, but I think the difference that you are seeing is that patients who have intestinal metaplasia in the esophagus (ie, Barrett's esophagus) receive surveillance, and that is why there is a slightly higher incidence of early tumors in that group of patients because there is a number that receive surveillance and are discovered, which is becoming a bigger number every year. The gastroenterologists in particular have not ascribed intestinal metaplasia limited to the GEJ as a premalignant condition that should be followed, and increasingly, there is evidence now that that is the precursor of adenocarcinoma of the GEJ. I personally believe those patients should be followed as well, and maybe we would discover some of these tumors before they present with dysphagia and more advanced tumors. Therefore I think the explanation for more T4 disease and the more advanced stages with GEJ tumors is they are not in a surveillance program in most circumstances. We could certainly try to do a more detailed matching of the 2 groups, but I am of the belief that the more statistics you have to use to prove something, the less likely it really is a significant difference. It came out as no difference with very easy statistics, and I believe that is the real situation.

Dr Rice. But your groups might not be completely comparable.

Dr DeMeester. I think they are perfectly comparable.

Dr Rice. We can agree to disagree.

Finally, in multivariable analysis Siewert and associates have shown that Siewert tumor type is not a predictor of survival, as you have found. Your analysis, however, does not include a multivariable analysis. Powerful support of your argument that type I and type II tumors are similar would be gained if a multivariable analysis of matched patients demonstrated that Siewert tumor type was not a significant variable predicting outcome. My fourth question is this: Will you include this important multivariable analysis in your report?

Dr DeMeester. Well, again, we could try to look at that, but to do a multivariable analysis, you have to have significant differences on univariate analysis. There was no difference really in the lymph node prevalence of metastases. There is no difference in survival by means of Kaplan-Meier/log-rank analysis. Therefore, again, although you and Dr Blackstone need to be commended, I think if you need a lot of statistics to prove a point, then there are some questions about how significant that point is. It was very clear that these 2 groups came out together. We can certainly do that, but, again, I do not see that we are going to change anything.

Dr Rice. Well, the way that would help you is if you prove that $\mathrm{T}, \mathrm{N}, \mathrm{M}, \mathrm{G}$, and number of lymph nodes are very, very important, as we all know.

Dr DeMeester. We know that, Tom. We know that. That has been proved over and over.

Dr Rice. But then you show that there is no room left to tell us that Siewert tumor type is significant, and you will help bury this archaic and not helpful classification.

Thank you.

Dr DeMeester. Thanks. We can certainly consider it.

Dr Mark J. Krasna.(Towson, $M d$ ) Tom, I have just a few questions regarding lymph nodes. Your colleague did a great presentation. Surprisingly, though, the overall prevalence of lymph node metastasis was roughly $50 \%$ in the chest and $50 \%$ in the abdomen. Therefore one question is this: Can you explain the lower incidence of lymph node involvement than seen in some of the other reported series, where it approaches $75 \%$ to $85 \%$ ? Was there a high use, for instance, of neoadjuvant therapy in your series?

Dr DeMeester. No. Neoadjuvant therapy was $18 \%$ in each group, and when we looked at node distribution and prevalence of lymph nodes, it was only in the en bloc group without neoadjuvant therapy so as not to confuse the issue of where nodes were and so forth.

Dr Krasna. Second, just to follow up on that, I was impressed that the celiac node involvement number was actually quite low. It was only about $10 \%$ in one group and a little bit more in the other. Is there an aversion in your group to include patients who have positive celiac nodes, or is that simply what your finding is at the time of your en bloc resection? The reason I ask is this. As we have heard from the proposed new staging system, celiac node involvement will be like any other node involvement in the future, and if there is an aversion to operate on such patients, there apparently is no clear-cut evidence that that is correct. Therefore I am curious about your philosophy.

Dr DeMeester. No, we have no aversion to that. In fact, we published years ago that the presence of celiac node involvement has absolutely no bearing on survival. If you look at celiac node involvement in the context of the number of involved nodes, you will find it is the number of involved nodes that predicts survival. The celiac node itself is meaningless. Therefore I would prefer to have 1 celiac node positive than to have 5 nonceliac nodes positive. I would take the first choice any day of the week. Therefore it is the number of involved nodes and not the distribution that is important. We have no qualms about taking out celiac nodal disease. 